\title{
Performance Analysis of 2-Step Random Access with CDMA in Machine-Type Communication
}

\author{
Jinho Choi
}

\begin{abstract}
There is a growing interest in the transition from 4-step random access to 2 -step random access in machine-type communication (MTC), since 2-step random access is well-suited to short message delivery in various Internet of Things (IoT) applications. In this paper, we study a 2-step random access approach that uses code division multiple access (CDMA) to form multiple channels for data packet transmissions with a spreading factor less than the number of channels. As a result, the length of data transmission phase in 2-step random access can be shorter at the cost of multiuser interference. To see how the decrease of the length of data transmission phase can improve the spectral efficiency, we derive the throughput as well as the spectral efficiency. From the results of analysis, we can show that the 2-step CDMA-based random access approach can have a higher spectral efficiency than conventional 2-step approach with orthogonal channel allocations, which means that the performance of MTC can be improved by successfully transmitting more data packets per unit time using CDMA. This is also confirmed by simulation results.
\end{abstract}

Index Terms-MTC; Code Division Multiple Access; Spectral Efficiency

\section{INTRODUCTION}

The Internet of Things (IoT) has been extensively studied to connect a large number of devices for various applications including smart home, smart cities, smart manufacturing, and so on [1] [2]. To support the connectivity for various IoT applications over a wide area, a number of different options can be considered [3]. Among those, in cellular IoT, machinetype communication (MTC) [4] [5] plays a crucial role in providing the connectivity for IoT devices and sensors through cellular systems [6] [7]. Due to sparse activity of those devices and sensors, MTC is usually based on random access to keep signaling overhead low [4] [5] [8] [9].

In MTC, a fraction of devices are active at a time and use random access to establish connections to transmit their data (e.g., random access channel (RACH) procedure in the longterm evolution advanced (LTE-A) systems [4]). For random access, a common pool of preambles is used [4] [5]. A device that has data packets to transmit, which is called an active device, randomly chooses a preamble from the pool and transmits it to a base station (BS) (through physical random access channel (PRACH) in $\mathrm{RACH}$ procedure) which is the first step of a handshaking process to establish connection in most MTC schemes (e.g., [4]). Due to multiple active devices that choose the same preamble, there exist preamble collisions and this step can be seen as contention-based access. There are

The author is with the School of Information Technology, Deakin University, Geelong, VIC 3220, Australia (e-mail: jinho.choi@deakin.edu.au). This research was supported by the Australian Government through the Australian Research Council's Discovery Projects funding scheme (DP200100391).
3 more steps in the handshaking process to allocate dedicated uplink (data) channels (which are physical uplink shared channel (PUSCH) in RACH procedure) to active devices so that they can transmit their data packets to the BS, which is the third step. Note that the 2nd and 4th steps are feedback transmissions from the BS to devices. The resulting random access approaches are called 4-step random access as there are 4 main steps.

Provided that messages transmitted from devices are short, to improve efficiency, 4-step random access can be replaced with 2-step random access [10], which is also referred to as grant-free random access or compressive random access (CRA) [11] [12] [13] [14] [15]. The first step consists of two stages, where an active device transmits a preamble and then a data packet. In other words, the first and third steps of 4step random access are combined into the first step of 2-step random access, while the second step is to send feedback signals from the BS to devices. In general, 2-step random access can be seen as a multichannel random access scheme, where each (multiple access) channel can be characterized by a preamble in the first stage of the first step. In the second stage of the first step, the preamble can be used as a spreading sequence to transmit data packet, which results in code division multiple access (CDMA) based random access [16] [17]. As shown in [15], it is also possible to use different spreading code (but associated with the preamble in the first stage) for spreading in the second stage.

If a BS is equipped with multiple antennas, based on the notion of massive multiple input multiple output (MIMO) [18], without spreading, the BS is able to decode data packets transmitted by multiple active devices in a shared channel simultaneously [19] [20] [21] [22]. Due to unbounded capacity of massive MIMO [23], it seems that massive MIMO is a solution to massive MTC. However, the cost of BSs with a large number of antenna elements may be high. Thus, for small cells, a BS equipped with single or a few antennas could be practical for MTC. As a result, in 2-step random access, for data transmissions in the second stage, CDMA or any orthogonal multiple access scheme such as time division multiple access (TDMA) has to be employed. When TDMA is used to support multiple data packet transmissions, the data transmission phase is to be divided into the same number of blocks as the number of preambles so that an active device that chooses the $l$ th preamble is to send its data packet through the $l$ th block in the 2nd stage. However, this may result in waste of resources if the number of active devices is less than that of blocks. To avoid it, in [24], the number of blocks is dynamically decided by the number of active devices. However, since this approach requires feedback after 
the preamble transmission, it is not qualified as a 2-step random access approach.

In this paper, we consider a 2-step random access scheme where CDMA is used for transmitting data packets from multiple active devices. In addition, a set of orthogonal preambles is assumed so that the channel state information (CSI) of each active device can be estimated without interference if there is no preamble collision. As in [15], it is assumed that each preamble is associated with a different spreading sequence ${ }^{1}$. In particular, the case that the length of spreading sequence is shorter than that of preambles is considered. As a result, the length of the second stage can be shorter, which can result in an improved spectral efficiency. However, unlike preambles, spreading sequences are not orthogonal (as their length is shorter than the number of them) and there exists multiuser interference. To mitigate multiuser interference, multiuser detection [25] [26] can be used under certain conditions. Since the performance depends on key parameters (e.g., the number of active devices, the number of preambles, and the length of spreading sequences), the throughput and spectral efficiency are derived in terms of those key parameters, which are the main contributions of the paper. From the analysis, we can show that 2-step random access with CDMA can outperform 2-step random access with orthogonal channel allocations, e.g., TDMA.

Note that preambles are used not only for the channel estimation, but also for user activity detection. Thus, preamble design is important, although we do not consider it (we only use a set of orthogonal preambles). Thus, there are various approaches for preamble design. For example, in [27], a preamble design is considered to allow efficient user activity detection. In [28], an approach to effectively increase the number of preambles is studied.

The rest of the paper is organized as follows. In Section II we present the system model for 2-step random access in MTC. CDMA is considered in Section III to form multiple channels for data packet transmissions by multiple devices simultaneously. We briefly discuss preamble detection with power control in Section IV and multiuser detection in Section $\mathrm{V}$. The main contribution of the paper is presented in Section VI, where the throughput and spectral efficiency are derived for 2-step random access with CDMA. Simulation results are presented in Section VII and the paper is concluded with some remarks in Section VIII

Notation: Matrices and vectors are denoted by upper- and lower-case boldface letters, respectively. The superscripts $\mathrm{T}$ and $\mathrm{H}$ denote the transpose and complex conjugate, respectively. The support of a vector is denoted by $\operatorname{supp}(\mathbf{x})$ (which is the number of the non-zero elements of $\mathbf{x}) . \mathbb{E}[\cdot]$ and $\operatorname{Var}(\cdot)$ denote the statistical expectation and variance, respectively. $\mathcal{C N}(\mathbf{a}, \mathbf{R})$ represents the distribution of circularly symmetric complex Gaussian (CSCG) random vectors with mean vector a and covariance matrix $\mathbf{R}$.

\footnotetext{
${ }^{1}$ It is noteworthy that the system model of this paper is similar to that in [15], while we present a detailed throughput analysis that was not addressed in [15].
}

\section{SySTEM MODEL}

In this section, we consider a two-step random access system that consists of one BS and a number of devices for MTC.

Compared with 4-step random access approaches [4] [5], 2-step random access, which is also referred to as grant-free random access, can be more efficient due to low signaling overhead when devices' have short messages. In 2-step random access, the first step consists of preamble and data transmission phases as illustrated in Fig 1. An active device is to choose a preamble from a pool of $L$ pre-determined preambles, denoted by $\left\{\mathbf{p}_{1}, \ldots, \mathbf{p}_{L}\right\}$, and transmit it in the preamble transmission phase. Throughout the paper, we assume that preambles are orthonormal, i.e.,

$$
\mathbf{p}_{l}^{\mathrm{H}} \mathbf{p}_{l^{\prime}}=\delta_{l, l^{\prime}}
$$

and the length of $\mathbf{p}_{l}$ is $L$, i.e., $\mathbf{p}_{l} \in \mathbb{C}^{L}$. Here, $\mathbb{C}^{n}$ represents the $n$-dimensional complex coordinate space.

A data packet is then transmitted in the data transmission phase. It is assumed that a time slot is used to transmit a preamble and a data packet. Since all the devices are synchronized in MTC, it is expected that the length of data packet is the same for all devices (or the length of data transmission phase is decided by the maximum length of data packet among all the devices).

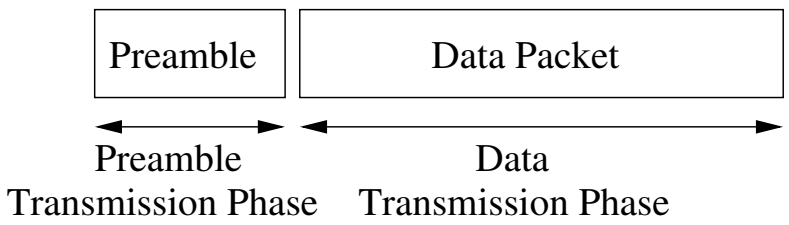

Fig. 1. Two phases (i.e., preamble transmission and data transmission phases) for a 2-step random access scheme.

The BS receives the signals from multiple active devices and performs preamble detection. That is, the BS is to detect transmitted preambles among the $L$ preambles. For each detected preamble, the BS can estimate the CSI of the associated active device and use it to decode the following data packet (via coherent detection).

In the second step, the BS broadcasts its decoding results to the devices with the feedback signal of acknowledgment (ACK) or negative acknowledgment (NACK).

Since the number of preambles, $L$, is limited, multiple active devices can choose the same preamble, which results in preamble collision. For a transmitted preamble by multiple active devices, the estimate of CSI becomes a noisy superposition of multiple channel coefficients. Thus, decoding associated with the transmitted preamble will fail. In other words, preamble collision leads to NACK to all the devices associated preamble collision.

\section{DATA PACKET TRANSMissions In TWO-STEP RANDOM ACCESS}

If there are multiple active devices, they send data packets simultaneously during the data transmission phase in 2-step random access. Thus, there should be multiple channels. In this 
section, we discuss two different approaches to form multiple channels for data transmission when the BS does not have a large number of antennas (we also briefly explain the case when the BS has a large number of antennas later in this section).

\section{A. Time Division Multiple Access}

In 2-step random access, the maximum number of channels for data packet transmissions is $L$, in which each channel is associated with one preamble. That is, an active device that chooses and transmits preamble $l$ is to send its data packet in data channel $l$. TDMA can be used for orthogonal channel allocations for data transmissions. In this case, the sub-slot for the data transmission phase is divided into $L$ time blocks and the resulting approach is referred to as the TDMAbased approach for convenience. For convenience, denote by $D$ the length of data packet of each active device for short message delivery. Then, the length of the data transmission phase becomes $D L$.

\section{B. Code Division Multiple Access}

There is a drawback of the TDMA-based approach. Suppose that the $l$ th preamble is not chosen by any active devices. Then, clearly, the $l$ th block becomes empty, which can result in a low spectral efficiency. Thus, in this paper, we study an approach based on CDMA for data packet transmissions, which is similar to the approach in [15].

Let $\mathbf{c}_{l}$ denote the spreading sequence of length $N$ associated with the $l$ th preamble. Here, $N$ is also called the spreading factor [29]. Unlike preambles, it is assumed that the $\mathbf{c}_{l}$ 's are not orthogonal as $N<L$ (i.e., the length of spreading sequences is shorter than that of preambles). The resulting approach is referred to as the CDMA-based approach. Since $N<L$, the length of the data transmission phase of the CDMA-based approach is shorter than that of the TDMAbased approach, which can improve the spectral efficiency at the cost of multiuser interference.

For convenience, however, we assume that the length of slot is the same for both TDMA and CDMA, which is denoted by $T_{\text {slot. }}$. In this case, since $N<L$, the number of data symbols in data transmissions becomes $\bar{D}=\frac{L D}{N}$ as illustrated in Fig. 2 Here, we have $T_{\text {slot }}=L D+L=L+N \bar{D}$.

There are a few remarks as follows.

- If $N=L$, we can use orthogonal spreading sequences (i.e., $\mathbf{c}_{l}=\mathbf{p}_{l}$ for all $l$ ). However, the resulting approach is equivalent to the TDMA-based approach (as each spreading sequence can be seen as an orthogonal block in the time domain). Thus, for the CDMA-based approach, we only need to consider $N<L$.

- With the same length of slots, the number of data symbols per slot in the CDMA-based approach, $\bar{D}$, is larger than that the TDMA-based approach, $D$, as long as $N<L$. In addition, as $\frac{L}{N}$ decreases, the CDMA-based approach becomes more efficient than the TDMA-based approach in terms of spectral efficiency. However, since decoding fails if $N$ is smaller than the number of active devices

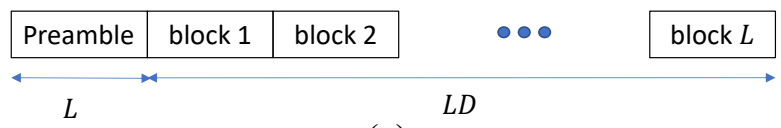

(a)

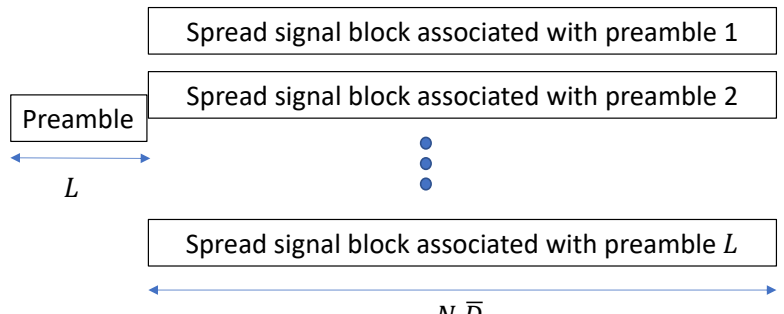

(b)

Fig. 2. Two different structures for the data transmission phase: (a) TDMAbased approach with the data transmission phase of length $D L$; (b) the CDMA-based approach with the data transmission phase of length $\bar{D} N$.

in the CDMA-based approach (as will be shown in Section $\mathrm{V}, N$ cannot be arbitrarily small. Thus, throughput will be analyzed in Section VI, to see the impact of $N$ on the performance.

- The length of slot cannot be long for coherent detection. As a result, 2-step random access would be preferable only when the length of message (i.e., $D$ or $\bar{D}$ ) is sufficiently short and the number of devices is not too large (to limit $L$ ). Thus, to support a large number of devices, multiple 2-step random access schemes should run in parallel over multiple resource blocks.

\section{With Massive MIMO}

If the BS is equipped with a large antenna array, the notion of MIMO can be exploited [18]. In particular, the BS can estimate the channel vectors using the bank of the correlators with $L$ preambles. Provided that there is one active device for a given preamble, the output of the correlator can provide an estimate of the channel vector of the active device. As the number of antennas increases, it is known that the channel vectors of different users or devices become asymptotically orthogonal. Thus, although all the active devices transmit their data packets through one data channel, the BS is able to decode them by exploiting a high spatial selectivity [19] [20] [21] [22]. In this case, the length of data transmission phase becomes $D$. Certainly, two-step random access with massive MIMO has a high spectral efficiency at the cost of a large antenna array, which is often infeasible for small BSs that support relatively small areas (i.e., small cells).

\section{Preamble Detection and Performance ANALYSIS}

In this section, we discuss preamble detection and show that reliable preamble detection can be achieved under power control. In the rest of the paper, we assume that the BS is equipped with a single antenna. Thus, no spatial diversity is to be exploited at the BS. 


\section{A. Preamble Detection with Power Control}

Suppose that there are $K$ active devices. Let $\mathcal{K}_{l}$ denote the index set of the active devices that choose preamble $l$. In addition, denote by $h_{k} \in \mathbb{C}$ the channel coefficient ${ }^{2}$ from the $k$ th active device to the BS. Then, the received signal at the BS during the preamble transmission phase is given by

$$
\begin{aligned}
\mathbf{y} & =\sum_{1=1}^{L} \mathbf{p}_{l} \sum_{k \in \mathcal{K}_{l}} h_{k} \sqrt{P_{k}}+\mathbf{n}_{1} \\
& =\sum_{k=1}^{K} \mathbf{p}_{l(k)} h_{k} \sqrt{P_{k}}+\mathbf{n}_{1} \in \mathbb{C}^{L},
\end{aligned}
$$

where $P_{k}$ stands for the transmit power of active device $k, l(k)$ is the index of the preamble that is chosen by active device $k$, and $\mathbf{n}_{1} \sim \mathcal{C N}\left(0, N_{0} \mathbf{I}\right)$ is the background noise vector.

Throughout the paper, it is assumed that the transmit power is decided according to the following power control policy:

$$
\left|h_{k} \sqrt{P_{k}}\right|^{2}=\left|h_{k}\right|^{2} P_{k}=P_{\mathrm{rx}},
$$

where $P_{\mathrm{rx}}$ represents the target receive power at the BS. In order to allow the power control in 2, it has to be assumed that any active device knows its channel coefficient $h_{k}$ prior to preamble transmission so that its power can be decided according to 22. To this end, we assume that the system is based on time division duplexing (TDD) so that active devices can estimate their channel coefficients using the channel reciprocity [30] when the BS sends a beacon or pilot signal that is to be transmitted periodically.

Furthermore, it is assumed that the coherence time is sufficiently long so that $h_{k}$ remains unchanged over the duration of slot, which might be a reasonably assumption when devices are not mobile.

Note that if the magnitude of the channel coefficient is not sufficiently large and the transmit power can exceed the maximum transmit power ${ }^{3}$ denoted by $P_{\max }$, i.e.,

$$
P_{k}=\frac{P_{\mathrm{rx}}}{\left|h_{k}\right|^{2}}>P_{\max }
$$

we assume that the device cannot transmit signals. If such a device transmits a preamble, the received signal does not have a sufficiently high SINR to allow reliable preamble detection, while it becomes an interfering signal to another active device that happens to choose the same preamble. Thus, the device that cannot meet (2) should not be active.

For convenience, let $h_{k}=\left|h_{k}\right| e^{j \theta_{k}}$, where $\theta_{k}$ is the phase of $h_{k}$. In this case, from (2), we have $h_{k} \sqrt{P_{k}}=\sqrt{P_{\text {rx }}} e^{j \theta_{k}}$. Since the preambles are orthonormal, the bank of $L$ correlators can be used to detect transmitted preambles. The output of the $l$ th correlator is given by

$$
\begin{aligned}
z_{l} & =\mathbf{p}_{l}^{\mathrm{H}} \mathbf{y}=\sum_{k \in \mathcal{K}_{l}} h_{k} \sqrt{P_{k}}+\mathbf{p}_{l}^{\mathrm{H}} \mathbf{n}_{1} \\
& =\sqrt{P_{\mathrm{rx}}} a_{l}+n_{l},
\end{aligned}
$$

\footnotetext{
${ }^{2}$ Throughout the paper, we consider a narrowband system. As a result, the channel from each device to the BS can be seen as a flat fading channel.

${ }^{3}$ Note that the norm of preambles is normalized, i.e., $\left\|\mathbf{p}_{l}\right\|=1$, which means that $P_{k}=P_{k}\left\|\mathbf{p}_{l(k)}\right\|^{2}$ is the total energy to transmit a preamble by an active device. Thus, although actual transmit power is limited, $P_{k}$ can be high if a longer preamble is used.
}

where $a_{l}=\sum_{k \in \mathcal{K}_{l}} e^{j \theta_{k}}$ and $n_{l}=\mathbf{p}_{l}^{\mathrm{H}} \mathbf{n}_{1}$. Thanks to orthonormal preambles, we have $n_{l} \sim \mathcal{C N}\left(0, N_{0}\right)$.

Provided that devices can estimate $h_{k}$ precisely based on the channel reciprocity, it is possible that each active device can compensate the phase of the signal to be transmitted. For example, the signal that active device $k$ transmits is $\sqrt{P_{k}} e^{-j \theta} \mathbf{p}_{l(k)}$. Then, $a_{l}$ in (3) with phase compensation becomes

$$
a_{l}=\sum_{k \in \mathcal{K}_{l}} e^{j \theta_{k}} e^{-j \theta_{k}}=\left|\mathcal{K}_{l}\right| .
$$

For convenience, define the index set of transmitted preambles as

$$
\mathcal{A}=\left\{l|| \mathcal{K}_{l} \mid \geq 1\right\}
$$

Due to the possibility that the same preamble can be chosen by multiple active devices, we have

$$
Q=|\mathcal{A}| \leq \min \{L, K\} .
$$

For convenience, $\bar{l}(q)$ denotes the $q$ th element of $\mathcal{A}$ or the index of the $q$ th detected preamble in ascending order, i.e., $\mathcal{A}=\{\bar{l}(1), \ldots, \bar{l}(Q)\}$, where $\bar{l}(1)<\ldots<\bar{l}(Q)$. For example, suppose that $L=4$ and $K=3$. The first and second active devices choose preamble 4 , and the third active device chooses preamble 1 . Then, at the $\mathrm{BS}$, if all the transmitted preambles are correctly detected, we have $Q=2$ and $\mathcal{A}=\{1,4\}$. That is, $\bar{l}(1)=1$ and $\bar{l}(2)=4$, while $l(1)=l(2)=4$ and $l(3)=1$. Note that if there is no preamble collision (which requires that $K \leq L$ ), we have $Q=K$ and there is a one-to-one relationship between $\{\bar{l}(q)\}$ and $\{l(k)\}$.

The ideal outcome of the preamble detection is to obtain $\mathcal{A}$. From $\left\{z_{l}\right\}$, hypothesis testing can be carried out to estimate $\mathcal{A}$ with and without phase compensation at devices.

\section{B. Hypothesis Testing and Performance Analysis}

1) With Phase Compensation: With phase compensation at active devices, $a_{l}$ is given in (4). Thus, the test statistic to detect the presence of preamble $l$ in $z_{l}$ becomes $\Re\left(z_{l}\right)$, which can be written as

$$
\Re\left(z_{l}\right)=\sqrt{P_{\mathrm{rx}}}\left|\mathcal{K}_{l}\right|+\Re\left(n_{l}\right),
$$

where $\Re\left(n_{l}\right) \sim \mathcal{N}\left(0, \frac{N_{0}}{2}\right)$.

Letting $\mathcal{H}_{0}$ and $\mathcal{H}_{1}$ represent the hypotheses of the absence and presence of preamble $l$, respectively, the following hypothesis testing can be considered:

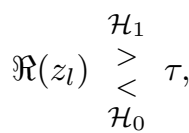

where $\tau>0$ is a decision threshold. Under $\mathcal{H}_{1}, \min \left|\mathcal{K}_{l}\right|=1$. Thus, we only consider the case that $\left|\mathcal{K}_{l}\right|=1$ for $\mathcal{H}_{1}$. Then, from (7), the probability of missed detection (MD) is given by

$$
\begin{aligned}
\mathbb{P}_{\mathrm{MD}} & =\operatorname{Pr}\left(\Re\left(z_{l}\right) \leq \tau|| \mathcal{K}_{l} \mid=1\right) \\
& =\mathcal{Q}\left(\sqrt{\frac{2}{N_{0}}}\left(\sqrt{P_{\mathrm{rx}}}-\tau\right)\right)
\end{aligned}
$$


On the other hand, the probability of false alarm (FA) is

$$
\mathbb{P}_{\mathrm{FA}}=\operatorname{Pr}\left(\Re\left(z_{l}\right) \geq \tau|| \mathcal{K}_{l} \mid=0\right)=\mathcal{Q}\left(\sqrt{\frac{2}{N_{0}}} \tau\right) .
$$

If $\tau=\frac{\sqrt{P_{\mathrm{rx}}}}{2}$, we have $\mathbb{P}_{\mathrm{MD}}=\mathbb{P}_{\mathrm{FA}}=\mathcal{Q}\left(\sqrt{\frac{P_{\mathrm{rx}}}{2 N_{0}}}\right)$. Clearly, with a sufficiently high signal to noise ratio (SNR), $\frac{P_{\mathrm{rx}}}{N_{0}}$, the probabilities of MD and FA become low, which implies that the preamble detection can be carried out at the BS reliably or $\mathcal{A}$ can be obtained with a high probability. For example, if the SNR is $10 \mathrm{~dB}, \mathcal{Q}\left(\sqrt{\frac{\mathrm{SNR}}{2}}\right)=0.0127$.

2) Without Phase Compensation: In TDD mode, due to the channel reciprocity, it is assumed that an active device can estimate the channel coefficient, $h_{k}$, for phase compensation. However, the radio frequency (RF) circuits on the BS differ from those on devices. Thus, the phase of $h_{k}$ may not be precisely estimated without calibration [31], while the power control in (2) can be carried out reliably as it is based on the magnitude, i.e., $\left|h_{k}\right|$. Consequently, the phase compensation may not be affordable at low-cost devices, and we now consider the hypothesis testing without phase compensation.

For simplicity, we only consider the case that $\left|\mathcal{K}_{l}\right|=1$ for $\mathcal{H}_{1}$. Then, under $\mathcal{H}_{1}, z_{l}$ is re-written as

$$
z_{l}=\sqrt{P_{\mathrm{rx}}} e^{j \theta_{l(k)}}+n_{l}
$$

For the hypothesis testing, the test statistic becomes $\left|z_{l}\right|^{2}$, which has the following distributions:

$$
\frac{2\left|z_{l}\right|^{2}}{N_{0}} \sim \begin{cases}\chi_{2}^{2}, & \text { under } \mathcal{H}_{0} \\ \chi_{2}^{2}\left(\frac{2 P_{\mathrm{rx}}}{N_{0}}\right), & \text { under } \mathcal{H}_{1},\end{cases}
$$

where $\chi_{n}^{2}$ represents the chi-squared distribution with $n$ degrees of freedom and $\chi_{n}^{2}(\mu)$ represents the noncentral chisquared distribution with $n$ degrees of freedom and noncentrality parameter $\mu$. In Fig. 3, we show the probabilities of MD and FA as functions of the decision threshold, $\tau$, when the SNR is $10 \mathrm{~dB}$. With a proper decision threshold, $\tau$, we can see that both the probabilities of MD and FA can be low.

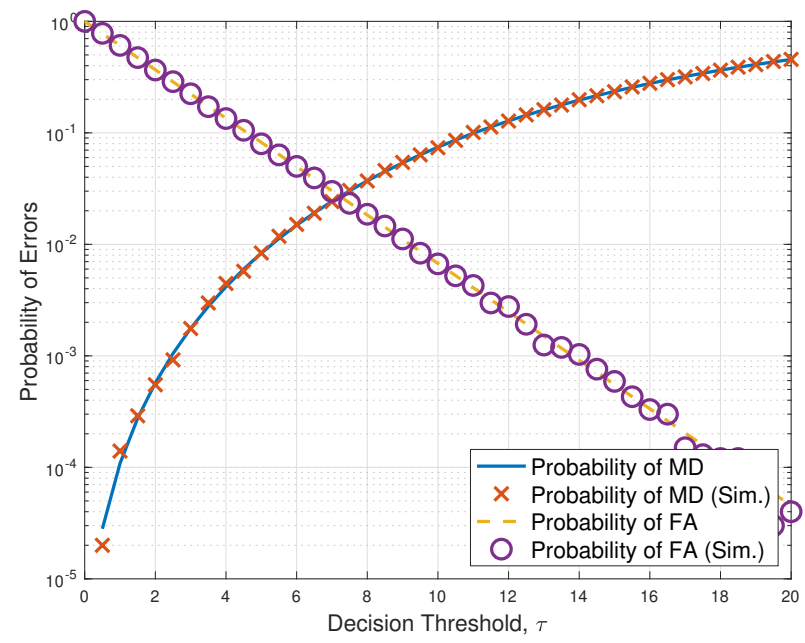

Fig. 3. The probabilities of MD and FA as functions of the decision threshold, $\tau$, when the SNR is $10 \mathrm{~dB}$.
Consequently, in the rest of the paper, we assume that the preamble detection can be reliably carried out, which allows the BS to obtain $\mathcal{A}$ with negligible errors.

\section{Multiuser Detection for CDMA Signals}

In this section, we discuss multiuser detection for the data transmission phase in the CDMA-based approach.

In the CDMA-based approach, an active device choosing preamble $l$ transmits a data packet spread by spreading sequence $l, \mathbf{c}_{l}$, during the data transmission phase. Thus, the received signal during the data transmission phase at the BS is given by

$$
\begin{aligned}
\mathbf{r}(t) & =\sum_{k=1}^{K} \mathbf{c}_{l(k)} h_{k} \sqrt{P_{k}} s_{k}(t)+\mathbf{n}_{2}(t) \in \mathbb{C}^{N} \\
& =\sum_{k=1}^{K} \overline{\mathbf{c}}_{l(k)} \sqrt{P_{\mathrm{rx}}} s_{k}(t)+\mathbf{n}_{2}(t), t=0, \ldots, D-1(12)
\end{aligned}
$$

where $\overline{\mathbf{c}}_{l(k)}=\mathbf{c}_{l(k)} e^{j \theta_{k}}$ without phase compensation or $\overline{\mathbf{c}}_{l(k)}=\mathbf{c}_{l(k)}$ with phase compensation, and $\mathbf{n}_{2}(t) \sim$ $\mathcal{C N}\left(0, N_{0} \mathbf{I}\right)$ is the background noise.

Suppose that the ideal outcome of the preamble detection is obtained. In other words, the BS knows $\mathcal{A}$. From $\mathcal{A}$, let

$$
\overline{\mathbf{C}}=\left[\begin{array}{lll}
\overline{\mathbf{c}}_{\bar{l}(1)} & \cdots & \overline{\mathbf{c}}_{\bar{l}(Q)}
\end{array}\right] \in \mathbb{C}^{N \times Q} .
$$

Then, $\mathbf{r}(t)$ is re-written as

$$
\mathbf{r}(t)=\overline{\mathbf{C}} \mathbf{x}(t)+\mathbf{n}_{2}(t)
$$

where $\mathbf{x}(t)=\left[\begin{array}{llll}x_{1}(t) & \ldots & x_{Q}(t)\end{array}\right]^{\mathrm{T}}$ and $x_{q}(t)=$ $\sum_{k \in \mathcal{K}_{\bar{l}(q)}} s_{k}(t)$. Using (14), the multiuser detection of $Q$ signals can be carried out. Suppose that $Q \leq N$. Then, with a sufficiently high $\mathrm{SNR}, \frac{P_{\mathrm{rx}}}{N_{0}}, \mathbf{x}(t)$ can be estimated reliably using a multiuser detection approach including linear detectors (such as the minimum mean squared error (MMSE) detector) [25] [26]. However, among $Q$ signals in $\mathbf{x}(t)$, the BS can only detect or decode the signals transmitted from the active devices without preamble collisions. Consequently, we expect that the number of successfully detected or decoded signals is less than or equal to $Q$, although $Q \leq N$.

On the other hand, if $Q>N$, (14) becomes an overloaded system and the BS can assume unsuccessful decoding as detection performance would be poor.

To see the detection performance when CDMA is used, we can consider a specific multiuser detector, namely the MMSE with lattice reduction (MMSE-LR) detector [26]. Suppose that quadrature phase shift keying (QPSK) is employed for data transmissions during the data transmission phase. In addition, for spreading sequences in the CDMA-based approach, we assume Alltop sequences [32] that have low cross-correlation. For a given $N$ (which is a prime greater than or equal to 5 ), there are $N^{2}$ Alltop sequences. In each run, $L$ out of $N^{2}$ sequences are randomly selected for the $L$ spreading sequences, $\left\{\mathbf{c}_{l}\right\}$. As mentioned earlier, the MMSE-LR detector is used for the multiuser detection.

Fig. 4 shows the bit error rate (BER) as a function of $\frac{E_{\mathrm{b}}}{N_{0}}$, where $E_{\mathrm{b}}$ represents the bit energy, when $L=20, N=11$, and $K=10$. Since QPSK is used, it is assumed that $E_{\mathrm{b}}=$ 
$P_{\mathrm{rx}} / 2$. With $K=10$, the following two different cases are considered:

1) $Q=8$ and 4 active devices with preamble collision;

2) $Q=9$ and 2 active devices with preamble collision.

From Fig. 4, we can see that a reasonably low BER (less than $10^{-1}$ ) is achieved for the active devices without preamble collision when $\frac{E_{\mathrm{b}}}{N_{0}} \geq 6 \mathrm{~dB}$. Note that the BER in Fig. 4 is uncoded BER. Thus, with channel coding, the packet can be reliably decoded.

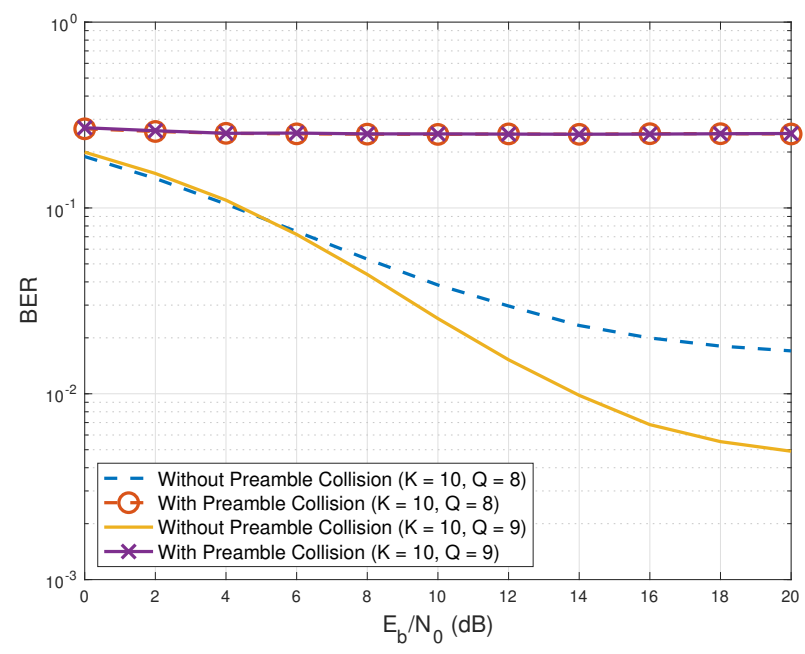

Fig. 4. BER of MMSE-LR detector as a function of $\frac{E_{\mathrm{b}}}{N_{0}}$ when $L=20$, $N=11$, and $K=10$.

Fig. 5 shows the BER and packet error rate as a function of the number of active devices, $K$, with one preamble collision when $L=20, N=11$, and $\frac{E_{\mathrm{b}}}{N_{0}}=20 \mathrm{~dB}$. For channel coding, a Bose-Chaudhuri-Hocquenghem (BCH) code of $(n, k, t)=(255,191,8)$ is considered, where $n, k$, and $t$ represent the code length, the number of message bits, and the error correction capability, respectively [33]. Since the CDMA-based approach has multiuser interference, the BER (of the active devices without preamble collision) increases with $K$. However, as long as $K$ is smaller than $N$, we see that both the uncoded BER and packet error rate are low. Note that in both Figs. 4 and 5 the BER of the active devices with preamble collision is high as expected, which means that their data packets cannot be decoded (as shown in Fig. 5 (b)).

Consequently, as long as $K$ is not too larger than $N$, we can assume that data packets transmitted from the active devices without preamble collision can be reliably decoded, while those with preamble collision cannot decoded. Based on this, the throughput could be found as in Section VI In the next subsection, we present the simulation results for the throughput and spectral efficiency.

\section{Throughrut AND Spectral EFFiciency ANALYsis}

In this section, we present the main results of performance analysis. In particular, we derive the throughput, which is defined as the average number of the active devices that do not have preamble collisions and their packets are successfully decoded if the condition of $Q \leq N$ is met for the CDMAbased approach, and the spectral efficiency.

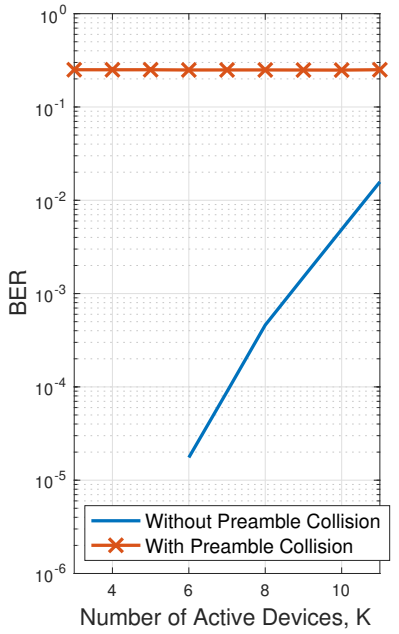

(a)

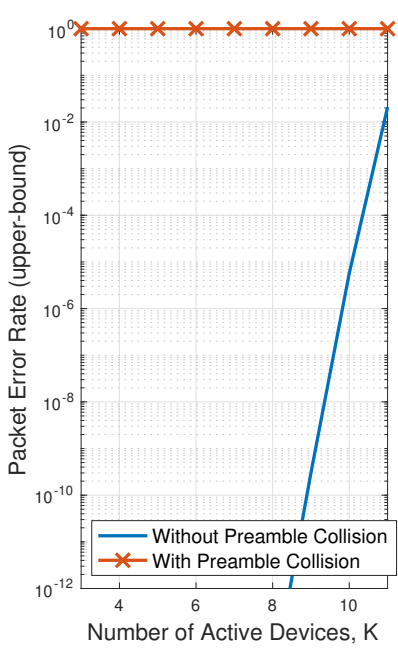

(b)
Fig. 5. Performance of MMSE-LR detector as a function of the number of active devices, $K$, with one preamble collision when $L=20, N=11$, and $\frac{E_{\mathrm{b}}}{N_{\mathrm{b}}}=20 \mathrm{~dB}$ : (a) uncoded BER; (b) packet error rate with BCH code of $(n, k, t)=(255,191,8)$.

\section{A. Throughput and Spectral Efficiency of TDMA}

In this subsection, for comparisons, we consider the TDMAbased approach, i.e., the case that $N=L$.

For convenience, define

$$
X_{k, l}= \begin{cases}1, & \text { if active device } k \text { chooses preamble } l \\ 0, & \text { o.w. }\end{cases}
$$

Let

$$
U_{l}=\mathbb{1}\left(\sum_{k=1}^{K} X_{k, l}=1\right) \in\{0,1\},
$$

where $\mathbb{1}(\cdot)$ represents the indicator function. Thus, the number of active devices without preamble collisions becomes $U=\sum_{l=1}^{L} U_{l}$. Clearly, with TDMA for orthogonal channel allocations, $U$ is also the number of active devices that can successfully transmit data packets.

If we assume that each active device randomly chooses one of $L$ preambles, it can be shown that

$$
\begin{aligned}
\alpha & =\operatorname{Pr}\left(U_{l}=1\right) \\
& =\left(\begin{array}{c}
K \\
1
\end{array}\right) \frac{1}{L}\left(1-\frac{1}{L}\right)^{K-1}=\frac{K}{L}\left(1-\frac{1}{L}\right)^{K-1} .
\end{aligned}
$$

Denote by $\kappa_{\mathrm{td}}(K)$ the number of signals that can be decoded with TDMA for $K$ active devices. Then, we have

$$
\kappa_{\mathrm{td}}(K)=\mathbb{E}[U]=\alpha L=K\left(1-\frac{1}{L}\right)^{K-1} .
$$

To consider the distribution of $K$, suppose that $M$ devices are uniformly distributed over a unit area. Furthermore, for a sufficiently large $M$, we assume that the number of active devices, $K$, follows a Poisson distribution with mean $\lambda=$ $M p_{a}$, where $p_{a}$ is the access probability. That is, the average number of active devices over the unit area is $\lambda$, which will 
be referred to as the activity intensity in the rest of the paper, and the probability that $K=k$ devices are active ${ }^{4}$ is

$$
\operatorname{Pr}(K=k)=\frac{\lambda^{k}}{k !} e^{-\lambda}
$$

Then, the throughput becomes

$$
\kappa_{\mathrm{td}}=\mathbb{E}\left[\kappa_{\mathrm{td}}(K)\right]=\lambda e^{-\frac{\lambda}{L}} .
$$

In addition, the spectral efficiency, which is the ratio of the number of successfully transmitted data symbols to the length of slot, is given by

$$
\eta_{\mathrm{td}}=\frac{D \kappa_{\mathrm{td}}}{T_{\text {slot }}}=\frac{D \lambda e^{-\frac{\lambda}{L}}}{L+L D}
$$

For a fixed $L$, as $D \rightarrow \infty$, we can see that that

$$
\lim _{D \rightarrow \infty} \eta_{\mathrm{td}}=\frac{\lambda}{L} e^{-\frac{\lambda}{L}} \leq e^{-1} .
$$

In other words, if the overhead of preamble becomes negligible, the spectral efficiency is upper-bounded by $e^{-1}$. However, $D$ may not be large for short message delivery in MTC. In addition, the length of slot, $L+L D$, has to be less than the coherence time for coherent detection at the BS. Thus, the overhead of preamble has to be taken into account, which lowers the spectral efficiency.

\section{B. Throughput and Spectral Efficiency of the Proposed Ap- proach}

In this subsection, we derive the throughput and spectral efficiency of the CDMA-based approach.

Prior to finding the throughput, we consider the probability of overloaded systems (i.e., $Q>N$ ). Let

$$
W_{l}=\mathbb{1}\left(\sum_{k=1}^{K} X_{k, l} \geq 2\right) \in\{0,1\} .
$$

It can be shown that

$$
\begin{aligned}
\beta & =\operatorname{Pr}\left(W_{l}=1\right) \\
& =1-\frac{K}{L}\left(1-\frac{1}{L}\right)^{K-1}-\left(1-\frac{1}{L}\right)^{K} .
\end{aligned}
$$

Furthermore, let $W=\sum_{l=1}^{L} W_{l}$. Recalling that $Q$ is the number of unique preambles transmitted, we have

$$
Q=U+W
$$

Thus, we need to find the distribution of $U+W$, which is the distribution of the number of bins with at least 1 ball when we throw $K$ balls into $L$ bins uniformly at random [34]. Since an exact distribution is not available, we may resort to an approximation. To this end, we need the following bound.

Lemma 1:

$$
\operatorname{Pr}(U+W \leq N) \geq \operatorname{Pr}(W \geq K-N) .
$$

Proof: If $U_{l}=1$, it implies that there is only one active device that transmits preamble $l$. In addition, if $W_{l}=1$, the

\footnotetext{
${ }^{4}$ In fact, $K$ follows the binomial distribution with parameters $M$ and $p_{a}$, i.e., $\operatorname{Pr}(K=k)=\left(\begin{array}{c}M \\ k\end{array}\right) p_{a}^{k}\left(1-p_{a}\right)^{M-k}$. However, for a sufficiently large $M,[19]$ is a good approximation [34].
}

number active devices that transmit preamble $l$ is greater than or equal to 2. Thus, since there are $K$ active devices, $\sum_{l} U_{l}+$ $2 \sum_{l} W_{l}$ has to be less than or equal to $K$, which results in

$$
U+2 W \leq K
$$

Then, by applying 27) to $\operatorname{Pr}(U+W \leq N)$, the bound in 26 can be obtained, which completes the proof.

If the $W_{l}$ 's are assumed to be independent (which is not true, but leads to a good approximation), we have

$$
\operatorname{Pr}(W \geq K-N \mid K>N) \approx \sum_{l=K-N}^{L}\left(\begin{array}{l}
L \\
l
\end{array}\right) \beta^{l}(1-\beta)^{L-l} .
$$

Thus, the probability of overloaded systems can be approximately given by

$$
\operatorname{Pr}(Q>N \mid K>N) \approx \sum_{l=0}^{K-N-1}\left(\begin{array}{l}
L \\
l
\end{array}\right) \beta^{l}(1-\beta)^{L-l} .
$$

Note that if $K \leq N, Q$ cannot be greater than $N$, i.e., $\operatorname{Pr}(Q>N \mid K \leq N)=0$. In addition, if $L$ is sufficiently large, the binomial distribution in 29 can be approximated by the Poisson distribution, which results in the following approximation of $\operatorname{Pr}(Q>N \mid K>N)$ :

$$
\begin{aligned}
\operatorname{Pr}(Q>N \mid K>N) & \approx \sum_{l=0}^{K-N-1} \frac{e^{-\nu} \nu^{l}}{l !} \\
& =\frac{\Gamma(K-N, \nu)}{(K-N-1) !},
\end{aligned}
$$

where $\nu=L \beta$ and $\Gamma(s, x)=\int_{x}^{\infty} t^{s-1} e^{-t} d t$ is the upper incomplete Gamma function.

For convenience, let $\kappa_{\mathrm{cd}}(K)$ denote the number of signals that can be decoded in the CDMA-based approach for a given $K$. If $K \leq N$, we have

$$
\kappa_{\mathrm{cd}}(K)=\mathbb{E}[U \mid K]=K\left(1-\frac{1}{L}\right)^{K-1} .
$$

On the other hand, if $K>N$, the throughput can be expressed as

$$
\kappa_{\mathrm{cd}}(K)=\mathbb{E}[U \mathbb{1}(Q \leq N) \mid K],
$$

because multiuser detection fails if $Q>N$. For a sufficiently large $L$, the inequality in (27) can be the following approximation $5+2 W \approx K$. Thus, $Q \leq N$ becomes $W \geq K-N$, which leads to the following approximate conditional throughput:

$$
\begin{aligned}
\kappa_{\mathrm{cd}}(K) & \approx \mathbb{E}[U \mathbb{1}(W \geq K-N) \mid K] \\
& \approx \mathbb{E}[U \mid K] \operatorname{Pr}(W \geq K-N \mid K), K>N
\end{aligned}
$$

where the second approximation is valid if $U$ and $W$ are independent (which is not true).

Note that we have $\operatorname{Pr}(W \geq K-N \mid K)=1$ if $K \leq N$ (since $W$ is a non-negative random variable). Thus, for a given

\footnotetext{
${ }^{5}$ If $W_{l}=1$, for a large $L$, it is likely that there are two active devices that choose preamble $l$. Thus, the inequality can be replaced with the equality with a high probability.
} 
$K$, from (31) and 33 , the conditional throughput for any $N \in\{1, \ldots\}$ can be given by

$$
\kappa_{\text {cd }}(K) \approx \alpha L \operatorname{Pr}(W \geq K-N \mid K) .
$$

Then, the average of the approximate throughput with 31] can be shown as follows:

$$
\begin{aligned}
\kappa_{\mathrm{cd}}= & \mathbb{E}\left[\kappa_{\mathrm{cd}}(K)\right] \\
= & \sum_{k=0}^{N} \omega_{L}(k) \operatorname{Pr}(K=k) \\
& +\sum_{k=N+1}^{\infty} \omega_{L}(k) \operatorname{Pr}(Q \leq N \mid K>N) \operatorname{Pr}(K=k) \\
= & \sum_{k=0}^{\infty} \omega_{L}(k) \operatorname{Pr}(K=k) \\
& -\sum_{k=N+1}^{\infty} \omega_{L}(k) \operatorname{Pr}(Q>N \mid K>N) \operatorname{Pr}(K=k),(35)
\end{aligned}
$$

where $\omega_{L}(k)=k\left(1-\frac{1}{L}\right)^{k-1}$. The approximation in 30) can be used to find a closed-form expression for the 2nd term on the right-hand side (RHS) in (35), which is

$$
\begin{aligned}
\text { 2nd term } & \approx \sum_{k=N+1}^{\infty} f(k) \sum_{l=0}^{k-N-1} \psi(l) \\
& =\sum_{q=0}^{\infty} \psi(q) \sum_{k=q}^{\infty} f(N+1+k),
\end{aligned}
$$

where

$$
\begin{aligned}
f(k) & =k\left(1-\frac{1}{L}\right)^{k-1} \operatorname{Pr}(K=k) \\
\psi(l) & =\frac{e^{-\nu} \nu^{l}}{l !} .
\end{aligned}
$$

In (36), since $\sum_{q} \psi(q)=1$, it can be seen that

$$
\begin{aligned}
\sum_{q=0}^{\infty} \psi(q) \sum_{k=q}^{\infty} f(N+1+k) & =\mathbb{E}\left[\sum_{k=Q}^{\infty} f(N+1+k)\right] \\
& \approx \sum_{k=\bar{Q}}^{\infty} f(N+1+k),
\end{aligned}
$$

where $Q$ is a random variable following the distribution, $\psi(q)$, and $\bar{Q}=\mathbb{E}[Q \mid K>N]$. Substituting (37) into (35), we have

$$
\begin{aligned}
\kappa_{\mathrm{cd}} & \approx \sum_{k=0}^{\infty} f(k)-\sum_{k=\bar{Q}}^{\infty} f(N+1+k)=\sum_{k=0}^{N+\bar{Q}} f(k) \\
& =\sum_{k=0}^{N+\bar{Q}} \omega_{L}(k) \operatorname{Pr}(K=k) .
\end{aligned}
$$

Then, for the distribution of $K$ in (19), after some manipulations, it can be shown that

$$
\kappa_{\mathrm{cd}} \approx \lambda e^{-\frac{\lambda}{L}} \underbrace{\frac{\Gamma(N+\bar{Q}, \bar{\lambda})}{(N+\bar{Q}-1) !}}_{=(b)} \leq \lambda e^{-\frac{\lambda}{L}}=\kappa_{\mathrm{td}}
$$

where $\bar{\lambda}=\lambda\left(1-\frac{1}{L}\right)$. The term (b) in 39 is the cumulative distribution function (cdf) of a Poisson random variable with mean $\bar{\lambda}$. Thus, if $N+\bar{Q}>\bar{\lambda}$, we expect that the term (b) can approach 1 , which means that the throughput of the CDMA-based approach can be close to that of the TDMAbased approach.

Note that since $\nu=L \beta$ and $\beta$ is dependent on $K$ as shown in 24], $\psi(l)$ is also a function of $k$, which is ignored in (36). That is, the last term in $\sqrt{36}$ is another approximation with a constant $\nu$ or $\beta=1-\frac{K}{L}\left(1-\frac{1}{L}\right)^{K-1}-\left(1-\frac{1}{L}\right)^{K}$, where $K=N+1$ (which is the smallest $K$ for $\nu$ in (30p). Thus, in (36), $\nu$ is replaced with

$$
\bar{\nu}=L-(N+1)\left(1-\frac{1}{L}\right)^{N}-L\left(1-\frac{1}{L}\right)^{N+1} .
$$

Thus, we have $\bar{Q}=\mathbb{E}[Q \mid K>N]=\bar{\nu}$ in (39).

From (39), the spectral efficiency of the CDMA-based approach is given by

$$
\eta_{\mathrm{cd}}=\frac{\bar{D} \kappa_{\mathrm{cd}}}{T_{\text {slot }}}=\frac{\bar{D} \lambda e^{-\frac{\lambda}{L}}}{L+N \bar{D}} \frac{\Gamma(N+\bar{Q}, \bar{\lambda})}{(N+\bar{Q}-1) !} .
$$

For fixed $L$ and $N$, as $\bar{D} \rightarrow \infty$, we can see that

$$
\lim _{\bar{D} \rightarrow \infty} \eta_{\mathrm{cd}}=\frac{\lambda e^{-\frac{\lambda}{L}}}{N} \frac{\Gamma(N+\bar{Q}, \bar{\lambda})}{(N+\bar{Q}-1) !} \leq \frac{L}{N} e^{-1} .
$$

Compared with 222, we can see that the upper-bound on the spectral efficiency of the CDMA-based approach in (42) can be higher than that of the TDMA-based approach by a factor of $\frac{L}{N}$. That is, in terms of the spectral efficiency, the performance of the CDMA-based approach can be $\frac{L}{N}$ times better than that of the TDMA-based approach.

Without using the upper-bounds for comparisons between the CDMA-based and TDMA-based approaches, the ratio of the spectral efficiencies can be directly considered. From 21) and 41, the performance gain over the TDMA-based approach with $N=L$ in terms of spectral efficiency can be obtained as

$$
\begin{aligned}
\varphi & =\frac{\eta_{\mathrm{cd}}}{\eta_{\mathrm{td}}} \\
& \approx \underbrace{\frac{L}{N}}_{=(a)} \underbrace{\frac{\Gamma(N+\bar{Q}, \bar{\lambda})}{(N+\bar{Q}-1) !}}_{=(b)}, N \leq L .
\end{aligned}
$$

As mentioned earlier the term (b) is close to 1 if $N+\bar{Q}>\bar{\lambda}$. Since the term (a) is greater than or equal to 1 , it is expected that $\varphi \geq 1$, i.e., the CDMA-based approach has a higher spectral efficiency than the TDMA-based approach.

Note that it is not necessary to consider the case that $N>$ $L$ (note that if $N=L$, as mentioned earlier, the CDMAbased approach becomes the TDMA-based approach). Clearly, $N>L$ is not useful as $N=L$ can avoid interference in packet transmissions from the active devices without preamble collisions. 


\section{Simulation Results}

In this section, a different set of simulation results are shown to see the throughput and spectral efficiency. For simulations, the Poisson arrival is assumed for $K$ unless $K$ is given.

In this subsection, we present the simulation results of the throughput and spectral efficiency of the CDMA-based approach, and compare them with those of the TDMA-based approach. All the simulation results are compared with the theoretical results in (39) and (41) for the throughput and spectral efficiency of the CDMA-based approach, respectively.

In Fig. 6, we show the throughput and spectral efficiency of the CDMA-based approach as functions of spreading factor, $N$, when $L=20$ and $\lambda=10$. Note that since the performance of the TDMA-based approach is independent of $N$, its throughput and spectral efficiency are constants as shown in Fig. 6 for comparisons. In Fig. 6 (a), it is shown that the throughput of the CDMA-based approach can approach that of the TDMA-based approach when $N \geq 13$. Thus, with a shorter length of the data transmission phase (as $N$ is less than $L$ ), it is possible to achieve the throughput of the TDMAbased approach, which results in a higher spectral efficiency. In Fig. 6 (b), it is clearly shown that the spectral efficiency of the CDMA-based approach can be higher than that of the TDMA-based approach when $N$ is close to $\lambda$.

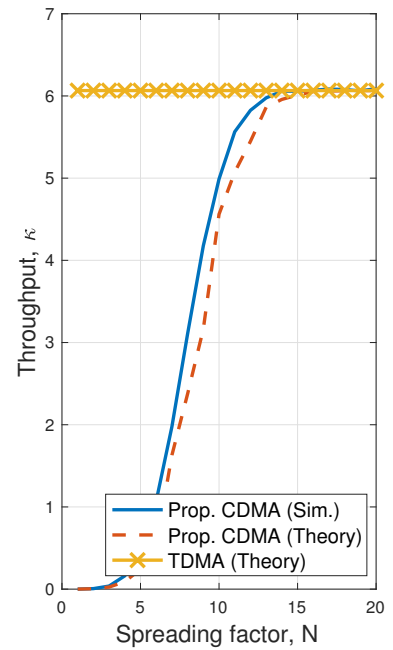

(a)

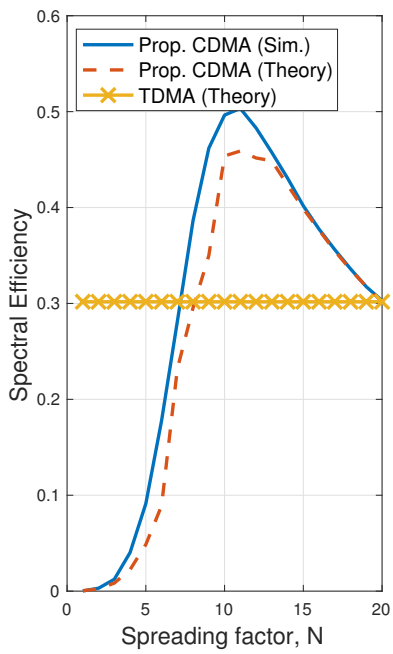

(b)
Fig. 6. Performance of the CDMA-based and TDMA-based approaches in terms of various values of spreading factor, $N$, when $L=20$ and $\lambda=10$ : (a) throughput versus $N$; (b) spectral efficiency versus $N$ with $D=200$.

In Fig. 7. we present simulation results for a larger system than that used in Fig. 6 by a factor of 5 . That is, we have $L=100$ and $\lambda=50$ in Fig. 7. By comparing Figs. 6 and 7 we can see that the performance gain of the CDMA-based approach becomes clearer for a larger system. In addition, in Figs. 6 and 7, we can see that the theoretical results are reasonably close to simulation results.

The throughput and spectral efficiency are shown as functions of $\lambda$ in Fig. 8 when $L=20$ and $N=10$. As shown in Fig. 8 (a), the throughput of the CDMA-based approach cannot be higher than that of the TDMA-based approach as $N$ is a half

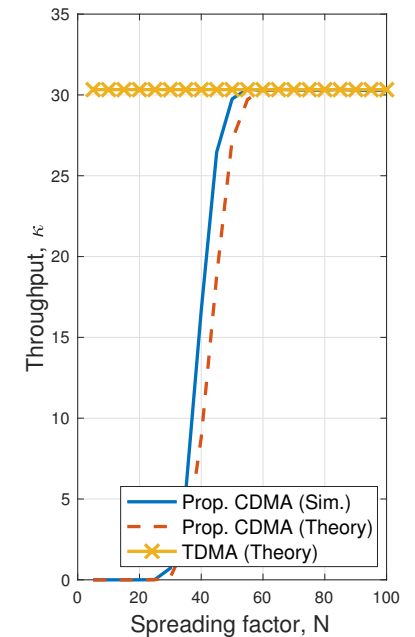

(a) (b)

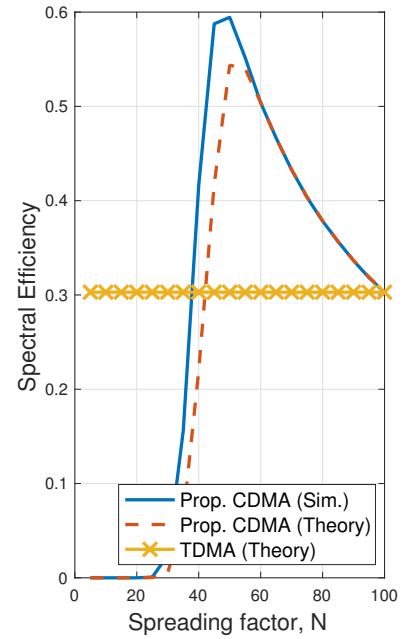

Fig. 7. Performance of the CDMA-based and TDMA-based approaches in terms of various values of spreading factor, $N$, when $L=100$ and $\lambda=50$ : (a) throughput versus $N$; (b) spectral efficiency versus $N$ with $D=2000$.

of $L$ for any values of $\lambda$. However, in Fig. 8 (b), it is shown that the spectral efficiency of the CDMA-based approach can be higher than the that of the TDMA-based approach.

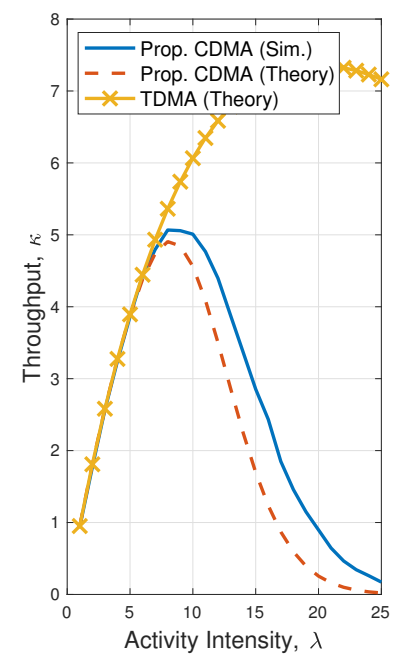

(a)

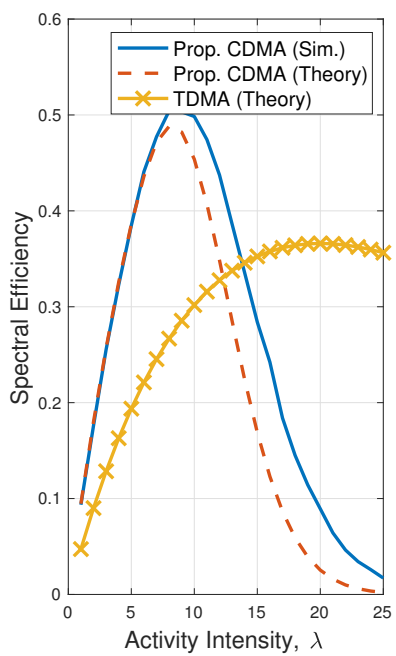

(b)
Fig. 8. Performance of the CDMA-based and TDMA-based approaches in terms of activity intensity, $\lambda$, when $L=20$ and $N=10$ : (a) throughput versus $\lambda$; (b) spectral efficiency versus $\lambda$ with $D=200$.

To see the impact of the length of preamble, $L$, on the performance in terms of the throughput and spectral efficiency, we present results when $N=20$ and $\lambda=15$ in Fig. 9 As $L$ increases, the probability of preamble collision decreases, which results in a higher throughput as shown in Fig. 9 (a). However, for the TDMA-based approach, with $D=10 L$, the length of slot grows quadratically with $L$. This leads to the decrease of the spectral efficiency as shown in Fig. 9(b). On the other hand, the spectral efficiency of the CDMA-based approach increases with $L$.

Fig. 10 shows the spectral efficiencies of the CDMA-based 


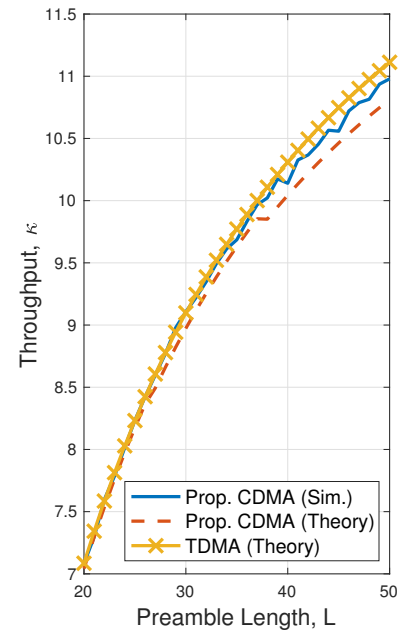

(a)

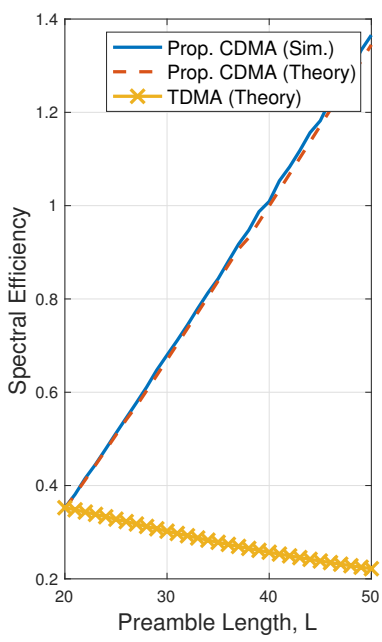

(b)
Fig. 9. Performance of the CDMA-based and TDMA-based approaches in terms of the length of preamble, $L$, when $N=20$ and $\lambda=15$ : (a) throughput versus $L$; (b) spectral efficiency versus $L$ with $D=10 L$.

and TDMA-based approaches as functions of the length of data packets, $D$, when $L=20, N=10$ and $\lambda=10$. It is shown that the spectral efficiency increases with $D$, but gets saturated with a sufficiently large $D$ (e.g., $D \geq 5 L$ ).

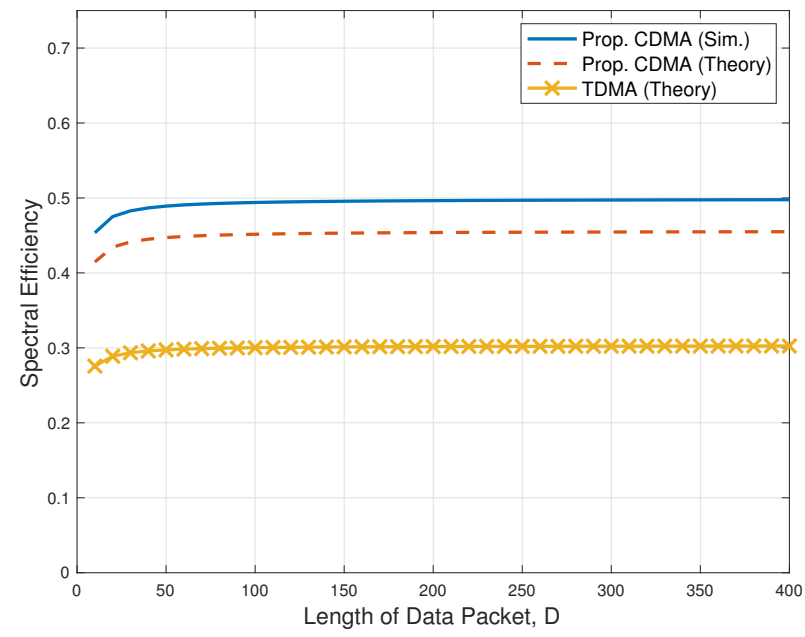

Fig. 10. Spectral efficiencies of the CDMA-based and TDMA-based approaches as functions of the length of data packets, $D$, when $L=20, N=10$ and $\lambda=10$

\section{CONCLUding REMARKS}

We studied a 2-step random access approach that uses CDMA to allow multiple devices to transmit their data packets simultaneously in this paper. Since the spectral efficiency can be improved with a spreading factor less than the number of spreading sequences, we derived the throughput and then spectral efficiency. From the analysis, it was shown that the 2-step random access approach with CDMA can have a higher spectral efficiency than the conventional 2-step random access approach with orthogonal channel allocations (e.g., TDMA). We also confirmed this with simulation results.
As assumed in the paper, the BS was equipped with a single antenna. Thus, the space domain was not exploited for multiple access. Since the space division multiple access (SDMA) can be combined with CDMA to support more devices, a generalization to the case that the BS is equipped with multiple antenna would be an interesting further research topic.

\section{REFERENCES}

[1] J. Gubbi, R. Buyya, S. Marusic, and M. Palaniswami, "Internet of Things (IoT): A vision, architectural elements, and future directions," Future Gener. Comput. Syst., vol. 29, pp. 1645-1660, Sept. 2013.

[2] J. Kim, J. Yun, S. Choi, D. N. Seed, G. Lu, M. Bauer, A. AlHezmi, K. Campowsky, and J. Song, "Standard-based IoT platforms interworking: implementation, experiences, and lessons learned," IEEE Communications Magazine, vol. 54, pp. 48-54, July 2016.

[3] J. Ding, M. Nemati, C. Ranaweera, and J. Choi, "IoT connectivity technologies and applications: A survey," IEEE Access, vol. 8, pp. 67646$67673,2020$.

[4] 3GPP TR 37.868 V11.0, Study on RAN improvments for machine-type communications, October 2011.

[5] 3GPP TS 36.321 V13.2.0, Evolved Universal Terrestrial Radio Access (E-UTRA); Medium Access Control (MAC) protocol specification, June 2016.

[6] H. Shariatmadari, R. Ratasuk, S. Iraji, A. Laya, T. Taleb, R. Jäntti, and A. Ghosh, "Machine-type communications: current status and future perspectives toward 5G systems," IEEE Communications Magazine, vol. 53, pp. 10-17, September 2015.

[7] C. Bockelmann, N. Pratas, H. Nikopour, K. Au, T. Svensson, C. Stefanovic, P. Popovski, and A. Dekorsy, "Massive machine-type communications in 5G: physical and MAC-layer solutions," IEEE Communications Magazine, vol. 54, pp. 59-65, Sep 2016.

[8] O. Galinina, A. Turlikov, S. Andreev, and Y. Koucheryavy, "Stabilizing multi-channel slotted ALOHA for machine-type communications," in Proc. IEEE ISIT, pp. 2119-2123, July 2013.

[9] J. Choi, "On the adaptive determination of the number of preambles in RACH for MTC," IEEE Communications Letters, vol. 20, pp. 13851388, July 2016.

[10] C. Bockelmann, N. K. Pratas, G. Wunder, S. Saur, M. Navarro, D. Gregoratti, G. Vivier, E. De Carvalho, Y. Ji, C. Stefanović, P. Popovski, Q. Wang, M. Schellmann, E. Kosmatos, P. Demestichas, M. RacealaMotoc, P. Jung, S. Stanczak, and A. Dekorsy, "Towards massive connectivity support for scalable mMTC communications in 5G networks," IEEE Access, vol. 6, pp. 28969-28992, 2018.

[11] H. F. Schepker, C. Bockelmann, and A. Dekorsy, "Exploiting sparsity in channel and data estimation for sporadic multi-user communication," in Proc. ISWCS 2013, pp. 1-5, Aug 2013.

[12] G. Wunder, P. Jung, and C. Wang, "Compressive random access for post-LTE systems," in Proc. IEEE ICC, pp. 539-544, June 2014.

[13] J. Choi and N. Y. Yu, "Compressive channel division multiple access for MTC under frequency-selective fading," IEEE Trans. Communications, vol. 65, no. 6, pp. 2715-2725, 2017.

[14] J. Choi, "Two-stage multiple access for many devices of unique identifications over frequency-selective fading channels," IEEE Internet of Things J., vol. 4, pp. 162-171, Feb 2017.

[15] A. T. Abebe and C. G. Kang, "Comprehensive grant-free random access for massive low latency communication," in 2017 IEEE International Conference on Communications (ICC), pp. 1-6, May 2017.

[16] H. Zhu and G. Giannakis, "Exploiting sparse user activity in multiuser detection," IEEE Trans. Communications, vol. 59, pp. 454-465, February 2011.

[17] L. Applebaum, W. U. Bajwa, M. F. Duarte, and R. Calderbank, "Asynchronous code-division random access using convex optimization," Physical Communication, vol. 5, no. 2, pp. 129-147, 2012.

[18] T. L. Marzetta, "Noncooperative cellular wireless with unlimited numbers of base station antennas," IEEE Trans. Wireless Communications, vol. 9, pp. 3590-3600, Nov. 2010.

[19] E. de Carvalho, E. Björnson, J. H. Sørensen, E. G. Larsson, and P. Popovski, "Random pilot and data access in massive MIMO for machine-type communications," IEEE Trans. Wireless Communications, vol. 16, pp. 7703-7717, Dec 2017.

[20] K. Senel and E. G. Larsson, "Device activity and embedded information bit detection using AMP in massive MIMO," in 2017 IEEE Globecom Workshops (GC Wkshps), pp. 1-6, Dec 2017. 
[21] L. Liu, E. G. Larsson, W. Yu, P. Popovski, C. Stefanovic, and E. de Carvalho, "Sparse signal processing for grant-free massive connectivity: A future paradigm for random access protocols in the Internet of Things," IEEE Signal Processing Magazine, vol. 35, pp. 88-99, Sept 2018.

[22] L. Liu and W. Yu, "Massive connectivity with massive MIMO-part I: Device activity detection and channel estimation," IEEE Trans. Signal Processing, vol. 66, pp. 2933-2946, June 2018.

[23] E. Bjornson, J. Hoydis, and L. Sanguinetti, "Massive MIMO has unlimited capacity," IEEE Trans. Wireless Communications, vol. 17, pp. 574-590, Jan 2018.

[24] J. Choi, "On throughput of compressive random access for one short message delivery in IoT," IEEE Internet of Things J., vol. 7, no. 4, pp. 3499-3508, 2020.

[25] S. Verdu, Multiuser Detection. Cambridge University Press, 1998.

[26] J. Choi, Optimal Combining and Detection. Cambridge University Press, 2010.

[27] L. Cheng, L. Liu, and S. Cui, "A covariance-based user activity detection and channel estimation approach with novel pilot design," in 2020 IEEE 21 st International Workshop on Signal Processing Advances in Wireless Communications (SPAWC), pp. 1-5, 2020.

[28] J. Choi, "An approach to preamble collision reduction in grant-free random access with massive MIMO," IEEE Trans. Wireless Communications, 2020 (accepted).

[29] A. J. Viterbi, CDMA: Principles of Spread Spectrum Communication. Addison-Wesley, 1995.

[30] G. S. Smith, "A direct derivation of a single-antenna reciprocity relation for the time domain," IEEE Trans. Antennas and Propagation, vol. 52, no. 6, pp. 1568-1577, 2004.

[31] M. Guillaud and F. Kaltenberger, "Towards practical channel reciprocity exploitation: Relative calibration in the presence of frequency offset," in 2013 IEEE Wireless Communications and Networking Conference (WCNC), pp. 2525-2530, 2013.

[32] W. Alltop, "Complex sequences with low periodic correlations (corresp.)," IEEE Trans. Information Theory, vol. 26, no. 3, pp. 350-354, 1980.

[33] S. B. Wicker, Error Control Systems for Digital Communication and Storage. Prentice Hall, 1995.

[34] M. Mitzenmacher and E. Upfal, Probability and Computing: Randomized Algorithms and Probability Analysis. Cambridge University Press, 2005. 